\title{
Multidrug-resistant and Extensively Drug-resistant Enterobacteriaceae: Prevalence, Treatments, and Outcomes: A Retrospective Cohort Study
}

Hadeel Alkofide ( $\nabla$ halkofide@ksu.edu.sa )

King Saud University College of Pharmacy https://orcid.org/0000-0002-5032-1895

Abdullah Alhammad

King Saud University

Alya Alruwaili

King Fahad Medical City

Ahmed Aldemerdash

King Saud University

Thamer Almangour

King Saud University

Aseel Alsuwayegh

King Saud University Medical City

Daad Almoqbel

Saudi Industrial Development Fund

Aljohara Albati

Saudi Food and Drug Authority

Aljohara Alsaud

Alfaisal University

Mushira Enani

King Fahad Medical City

\section{Research}

Keywords: Multi-Drug Resistant Bacteria, Extended-Drug Resistant Bacteria, Enterobacteriaceae

Posted Date: September 18th, 2020

DOI: https://doi.org/10.21203/rs.3.rs-75467/v1

License: (c) (i) This work is licensed under a Creative Commons Attribution 4.0 International License.

Read Full License 


\section{Abstract}

Background. Highly resistant gram-negative bacteria (GNB) is a global public health threat, especially in intensive care units (ICU). The purpose of this study is to explore the prevalence of drug resistant Enterobacteriaceae infections at an Intensive Care Unit (ICU) in Saudi Arabia. It also aims to assess the appropriateness of therapies used and whether these therapies improved clinical outcomes.

Methods. A retrospective study was conducted from 2015 to 2018 in a tertiary hospital ICUs in Saudi Arabia. Positive cultures for multidrug-resistant (MDR), extensive drug resistant (XDR), and pan drugresistant (PDR) Enterobacteriaceae: Klebsiella pneumoniae (K. pneumonia), Escherichia coli (E. coli), and Enterobacter species were included. Primary outcome was microbiological cure and 30 days in hospital mortality rates; while secondary outcome was length of hospital stay (LOS). Regression models were used to assess the relationship between appropriateness of therapy and outcomes.

Results. This study included 227 Enterobacteriaceae cultures in which $60 \%$ were either MDR $(n=130)$ or XDR $(n=8)$ infections; no PDR Enterobacteriaceae cultures were identified. The average subjects' age was $60.1 \pm 17.7$ years and $54 \%$ were females. Half of the MDR/XDR cultures were $E$. coli, followed by $33 \% K$. pneumoniae, and $16 \%$ Enterobacter infection. The most common antibiotics used were piperacillin/tazobactam (53\%), followed by carbapenems (47\%) and cephalosporins $(21.3 \%)$. Antibiotic therapy was considered appropriate in 85 out of 138 (61.59\%) subjects only. In-hospital death was $84 \%$, microbiological cure rate was achieved in $40 \%$ of cases, and the average LOS was 27 days. Appropriateness of antibiotic therapy prescribed was not a predictor of any of the study clinical outcomes.

Conclusion. In this study, there was a high prevalence of resistant Enterobacteriaceae infections, which were associated with a high mortality rate. This warrants the need to assess the effectiveness of antimicrobial stewardship program and infection prevention and control practices particularly in critically ill patients.

\section{Background}

Infection due to gram-negative bacilli (GNB) is a growing worldwide concern mainly due to hospitalacquired antibiotic resistant infection. $(1,2)$ Multidrug-resistant organisms (MDROs) are labeled as such because of their in vitro resistance to more than two antimicrobial agents. (2) According to the Center for Disease Control and Prevention (CDC), more than $70 \%$ of the bacteria that cause hospital-acquired infections are resistant to at least one antimicrobial agent, which is commonly used to treat them. (3) Enterobacteriaceae, Pseudomonas aeruginosa, and Acinetobacter baumannii are the most common resistant GNB acquired in hospital settings. (4) There are three types of antimicrobial resistance, multidrug resistance (MDR), which is acquired non-susceptibility to at least one agent in three or more antimicrobial classes. (2) Extensive drug-resistance (XDR), known as non-susceptibility to at least one agent in all but two or fewer antimicrobial classes (i.e., bacterial isolates remain susceptible to only one 
or two categories). (2) Finally, pan-drug resistance (PDR) is non-susceptibility to all agents in all antimicrobial classes. (2)

Globally, rates of MDR-GNB infections in intensive care units (ICUs) for which the treatment options are limited are increasing. (4-6) Critically ill patients are highly vulnerable to these infections due to multiple factors, including the use of mechanical ventilators and prolonged administration of antibiotics. (7) The likelihood of appropriate antimicrobial therapy decreases as resistance rates among GNB increases. (89) Studies report resistant organisms to colistin, a last resort antibiotic used to tackle many resistant GNB infections. (10) Unfortunately, the development of new antibacterial agents is not going in the same pace as the increasing rate of resistant organisms. (11)

The impact of the MDR-GNB infections can be determined from analyzing clinical outcomes, for example, hospital death rates, and the length of stay (LOS), either in the hospital or in the ICU. (12) Nevertheless, the association of resistant GNB with a prolonged hospital LOS and mortality remains controversial. (1316) While some studies did show a direct association between MDR-GNB and mortality, others were unable to find a relationship between the resistant GNB and either hospital LOS or mortality. (13-16) The reason it is believed that resistant bacteria are more likely to be associated with higher mortality rates is the possibility that appropriate antibiotic therapy is delayed compared to infections caused by antibioticsusceptible bacteria. (17)

Resistance to GNB differs from one place to another. Generally, there are limited data on the prevalence, treatments, and clinical outcomes of resistant GNB infections in ICUs worldwide and in Saudi Arabia. Therefore, we aim to describe the prevalence of MDR, XDR, and PDR Enterobacteriaceae infections including Klebsiella pneumoniae (K. pneumoniae), Escherichia coli (E. coli), and Enterobacter species in intensive care settings, list the empirical therapies used and whether they were appropriate or not, and report the microbiological cure rate, ICU length of stay, and 30-days mortality rate.

\section{Method}

\section{Study Design and Setting}

A retrospective cohort study was conducted at adult medical, surgical, and cardiac ICUs of King Saud University Medical City (KSUMC), a tertiary-care teaching hospital, located in Riyadh, Saudi Arabia. Data were collected from medical records starting from June 2015 until January 2018.

\section{Population}

Consecutive hospitalized adult patients who were admitted to the ICUs during the study period. All positive cultures of GNB Enterobacteriaceae, including K. pneumoniae, E. coli, and Enterobacter species were identified from Microbiology lab, then retrospectively classified as MDR, XDR or PDR regardless of the infection site and infection acquisition site. The study was approved by the institutional review board of King Saud University (Institutional review board number E-18-2874). 


\section{Data Collection}

Information collected included age, gender, either MDR, XDR or PDR infection, source of infection, type of bacteria, APACHE II score, use of antibiotics within the previous four weeks and 90 days, presence of a urinary catheter, mechanical ventilation, dialysis, presence of comorbid conditions (such as cardiovascular disease, lung disease, diabetes mellitus, solid tumors or hematological malignancy, liver disease, and renal failure). Lastly, information on ICU LOS, microbiological cure rate, empirical regimens, and 30-day mortality were collected.

\section{Definitions}

\section{Bacterial Resistance}

The definition of MDR, XDR, and PDR strains was based on the CDC and the European Centre for Disease Prevention and Control (ECDC) standardized international terminology. (2) Extended-spectrum $\beta$ lactamases (ESBLs)-producing organisms were defined if they confer resistance to most $\beta$-lactams antibiotics including penicillin, cephalosporins, and aztreonam. Carbapenemase -producing Enterobacteriaceae (CPE) was defined as resistant to imipenem, meropenem, doripenem, or ertapenem, or documentation that the isolate possesses a carbapenemase.

\section{Treatment Regimen}

Antibiotic therapy was categorized based on the number of medications used to monotherapy or combination therapy. Therapy was defined as appropriate if the patient received at least one antimicrobial agent to which the causative microorganisms were susceptible to and administered within 24 hour of culture collection. This definition was adapted from previous studies assessing the antimicrobial use in subjects infected with resistant strains of GNB.(9)

\section{Clinical Outcomes}

The microbiological cure rates were defined as the absence of the bacteria in a culture obtained from the same infection source. All-cause mortality was collected as documented in medical records during a 30day period, and LOS was calculated based on the number of days the patient was hospitalized in the ICU from the culture date.

\section{Microbiological Procedures}

Pathogen identity and antibiotics susceptibilities were determined by automation using one of two machines: Vitek 2 compact system or Microscan walkaway 96 plus, they show organism's identity with the percentage of assurance and susceptibility with 15-20 drugs (sensitive, intermediate or resistant), sometimes in case of CPE, PCR GeneXpert could be used. This is according to the recommendations of The Clinical and Laboratory Standards Institute (CLSI), Canadian AST guideline, and European EuCast.

\section{Statistical Analysis}


Frequencies and percentages were used to describe the categorical variables (e.g. gender, co-existing chronic conditions, type of bacteria, and source of infection). Means and standard deviations, or median and interquartile range (IQR) were used to describe continuous variables (age, and LOS). Chi-square and Fisher's exact tests were used to examine the factors related to bacterial mortality. Univariate and multiple regression analysis was conducted to identify variables associated with clinical outcomes (i.e., microbiological cure rate, 30-days mortality, and LOS) each in a separate model. In the multiple regression model, we controlled for variables that were statistically significant in univariate analyses with a p-value of $\leq$ 0.1. All statistical analyses were performed using $R$, version 3.5.0.

\section{Results}

\section{Study Population}

A total of 227 Enterobacteriaceae cultures were identified during the study period. Out of these, 130 (57.3\%) were MDR cultures, 8 (3.5\%) were XDR, and no PDR cultures were isolated. From the total MDR/XDR cultures identified, 116 out of 138 patients died during their hospital stay (84.1\%). The mean age and standard deviation (SD) of the study population was $60.1 \pm 17.7$ years, and almost $54 \%$ were females. About half of the subjects had a history of diabetes and $19.6 \%$ had a previous or active diagnosis of cancer. The most prevalent bacteria were $E$. coli, which was present in $51.4 \%$ of the subjects, followed by $K$. pneumonia in about $32.6 \%$ of subjects, and Enterobacter species in $15.9 \%$ of subjects. Seventy-one $(51.4 \%)$ of the total cultures were ESBL producers and $14(10.1 \%)$ were CPE. Urine was the most common source of infection. Most subjects were on ventilators $(61.6 \%)$ and used antibiotics four weeks $(71.7 \%)$ and 90 days $(79.7 \%)$ and prior to enrollment. When divided by survival status, none of the subject's clinical characteristics differed significantly between the groups except for gender, as the majority of those who survived were females. These results are summarized in Table 1.

\section{Empirical Therapy}

The majority of MDR cultures were treated with one agent (52\%), followed by combination therapy (30\%), and no therapy was given in $18 \%$ of the subjects (Figure 1). In those who received no antibiotics (25 out of 138 subjects), the site of infection was the urine in 10 subjects. Among those who received monotherapy, the most used antibiotic was piperacillin/tazobactam $(n=31,46 \%)$. In contrast, carbapenem plus aminoglycoside was the most frequent combination therapy used for MDR infections. Most XDR infections were treated with one agent $(n=6,75 \%)$, with carbapenem being used in half of them (Figure 2). For the two patients who received combination therapy, one was on carbapenem plus an aminoglycoside, while the other was on carbapenem and a fluoroquinolone.

In terms of appropriateness of therapy, among all study subjects ( $n=138)$, antibiotic therapy was considered appropriate in 85 (61.59\%) subjects only. From the MDR group, 83 out of 130 subjects received appropriate antibiotic therapy, compared to only two out of eight participants in the XDR group (Figure 1 and Figure 2). A detailed description of the antibiotics received in all study participants, and their appropriateness are listed in Tables 1 and 2 in the supplementary material. 


\section{Clinical Outcomes}

Out of 130 subjects with MDR infections, 108 (83\%) died at the end of follow up, 52 (40\%) reached microbiological cure, and the median LOS was 14 (28) days. Out of 8 subjects in the XDR group, 7 (79\%) died, microbiological cure achieved in 4 (50\%), and the median LOS was 38.5 (20.3) days, as shown in Table 2. Only male gender was significantly associated with mortality in univariate regression analysis (Table 3), while prior 90 days antibiotic use was related to lower odds of microbiological cure rate (Table 4). Since no other variables were found to be associated with clinical outcomes at a p-value $<0.1$, we were unable to perform multivariate regression models (Tables 3 and 4).

\section{Discussion}

The study estimated the prevalence rate of resistant Enterobacteriaceae infections at ICUs in Saudi Arabian tertiary hospital, described the antibiotic regimens used for these infections and identified factors associated with 30 -day mortality and microbiological cure rate. Around $60 \%$ of Enterobacteriaceae isolated from the ICU during the study period were either MDR or XDR, while it was not feasible to identify any PDR infections. These prevalence rates are generally higher than those reported in some studies across the globe. A study from India found lower rates of resistant GNB, in which they reported that $33.5 \%$ of bacterial isolates in a tertiary care hospital are MDR strains, $12.1 \%$ XDR and no PDR strains were found.(18) In 2019, a nationwide report from the United States reported the proportions of MDR Enterobacteriaceae isolates in inpatients to be 6.6\%.(19) Locally, one study on E. coli isolates only, has found the prevalence of MDR infections to be 67\%.(20) A driving factor for the higher rates seen in this study is the critically ill nature of the subjects, compared to previous report on inpatients which included ICU and non-ICU patients.(18-20) Other studies on subjects in the ICU, show either lower or similar rates of GNB resistance to the current study.(21-22) A study in Nepal on 137 patients, about $46 \%$ of GNB isolates at an ICU, were MDR infections.(21) On the other hand, a study from India, which looked at bacterial isolates in critically ill subjects with ventilator-associated pneumonia, found that $88 \%$ of those were GNB, in which $72 \%$ were MDR infections.(22)

There are a few significant differences between the current study and previously published reports regarding the prevalence of resistant Enterobacteriaceae. First, here there is a focus on Enterobacteriaceae species rather than GNB in general. According to the World Health Organization (WHO), Enterobacteriaceae have been considered to be critical and of priority number 1 of WHO priority pathogen list for research, discovery, and development of new antibiotics.(23) Second, both MDR and XDR cases are described here, compared to previous studies that focused on MDR infections alone. Lastly, studying subjects in the ICU settings is unique to the current analysis as limited data is available in the prevalence of these infections in critically ill subjects.

When looking specifically at ESBLs producing bacteria, the prevalence rate was $51.4 \%$, which is much higher than rates reported in other regions. For instance, in East Europe, the prevalence of ESBLs was over $10 \%$, while in Canada, it was 3.5\%. (24-25) Within the Arabian Gulf region, the prevalence ranged from as 
low as 7.5\% in Kuwait to as high as $41 \%$ in the United Arab Emirates.(26-27) Furthermore, other studies in Saudi Arabia reported ESBL detection to be between 22-36\% in Enterobacteriaceae.(28) In this study, the prevalence of CPE was around 6.2\%, which is higher than that reported in other studies.(29-33) This could be due to the high number of MDR-GNB infections observed in the study sample.

More than half of the subjects in the current study received an initial monotherapy for the treatment of their infections, with piperacillin/tazobactam and carbapenems being the most used agents. However, only about $62 \%$ of the prescribed antibiotics were deemed appropriate. Furthermore, around $18 \%$ of subjects did not receive any empirical antimicrobial therapy within the first 24 hours of culture results. It is important to highlight that in less than half of these subjects the primary site of infection was the urine, which might have been colonization, therefore no antibiotics were given. Limited studies have looked at the appropriateness of therapy in subjects with MDR and XDR infections in the ICU. One study in Italy has reported that $61 \%$ of therapies prescribed for MDR infections did not meet their criteria of appropriateness.(34) In a review article, focused explicitly on CPE infections compiling data from 889 patients, of which $48.6 \%$ received combination therapy, and $38.1 \%$ received monotherapy showed that only $11.3 \%$ of participants received inappropriate therapy.(35) However, due to the lack of a universally accepted definition of appropriate definitive antibiotic therapy against MDR and XDR pathogens in the literature, it is not easy to compare the results of the current study with the existing literature.

The case fatality rate due to MDR and XDR Enterobacteriaceae in the current study was $84 \%$, which is higher than that reported in previous literature. A study on hospitalized subjects found that the case fatality rate in MDR gram-negative isolates was around 34\%.(36) On the other hand, Blot et al. studied 328 patients with GNB in an ICU setting and found that in MDR infections there was a $45 \%$ case fatality rate.(37) The current study differs from these previous studies in that there is a focus only on critically ill subjects, and the inclusion of both MDR and XDR Enterobacteriaceae infections which could have accounted for the higher overall mortality in the study population. In this study, important risk factors for predicting mortality in subjects with MDR and XDR Enterobacteriaceae were assessed, such as previous antibiotic use and resistance type (i.e., ESBL and CPE). However, only the male gender was found to affect mortality rates significantly.

This study has several limitations, including being a single-center study, in which the results might be affected by the practice of this center, in addition to limiting generalizability. However, the ICU setting is part of a large tertiary hospital that accepts patients from any part of Saudi Arabia. Furthermore, information on antibiotics administered beyond the first 24 hours of positive culture results were not collected. Whether the positive cultures were colonization rather than active infections, specifically those in the urine was not clear, which might have affected the definition of appropriate antibiotic therapy. Also, this is a retrospective analysis of clinical data; therefore, there could be missing data, nevertheless, we attempted to collect all study-related information for all participants. Lastly, we have a relatively small sample of subjects, limiting the ability to perform adjusted regression analyses. Nevertheless, this study has several strengths. First, both MDR and XDR infections were reviewed in this study. Second, 
information on important clinical outcomes were collected, specifically mortality rates. Finally, detailed description of the use of antibiotics within the first 24 hours of culture results were presented.

\section{Conclusions}

The rate of antimicrobial resistance among GNB differs significantly worldwide and rapidly changing over time. There is limited data regarding the prevalence of MDR, XDR, and PDR Enterobacteriaceae in Saudi Arabia. Most local studies reported the prevalence as ESBL and carbapenemase producers, which may owe to a lack of standard definitions. This study shows that more than half of Enterobacteriaceae infections in critically ill subjects are due to resistant bacteria, with $E$. coli being the most common pathogen. The high mortality rate observed in this cohort is alarming, and further research on a larger sample of subjects from different centers is needed to find possible predictors of mortality. Finally, global efforts need to focus on developing new antimicrobial agents that can minimize the negative clinical and economic consequences of antibiotic-resistant organisms.

\section{Abbreviations}

CDC

Disease Control and Prevention

CLSI

Clinical and Laboratory Standards Institute

CPE

Carbapenemase -producing Enterobacteriaceae

ECDC

European Centre for Disease Prevention and Control

ESBLs

Extended-spectrum $\beta$-lactamases

GNB

Gram-negative bacilli

ICU

Intensive care unit

IQR

Interquartile range

KSUMC

King Saud University Medical City

LOS

Length of stay

MDROs

Multidrug-resistant organisms

MDR 
Multidrug resistance

PDR

Pan-drug resistance

SD

Standard deviation

WHO

World Health Organization

XDR

Extensive drug-resistance

\section{Declarations}

\section{Ethics approval and consent to participate}

The study was approved by the institutional review board of King Saud University (Institutional review board number E-18-2874). All data were and consent waiver was obtained for the study.

\section{Consent for publication}

Not applicable.

\section{Availability of data and materials}

The datasets used and/or analysed during the current study are available from the corresponding author on reasonable request.

\section{Competing interests}

The authors declare that they have no competing interests.

\section{Funding}

This research project was supported by a grant from the "Research Center of the Center for Female Scientific and Medical Colleges”, Deanship of Scientific Research, King Saud University.

\section{Authors' contributions}

HA and AA both made equal and substantial contributions to the design, conception, data collection and acquisition, analysis and drafting of the manuscript. AA, AA, TA, and ME participated in the study design and provided supervision and assistance towards data analysis, interpretation, and critically revising the manuscript. AA, DA, AA, AA, have performed data collection, assisted in data analysis, and provided feedback on the manuscript. All authors read and approved the final manuscript

\section{Acknowledgements}


We thank Undergraduate Research Support Program, for their support and KSUMC microbiology lab for their massive support and help. This research project was supported by a grant from the "Research Center of the Center for Female Scientific and Medical Colleges”, Deanship of Scientific Research, King Saud University.

\section{Conflict of Interests}

The authors declare that there is no conflict of interests regarding the publication of this paper.

\section{References}

1. Exner M, Bhattacharya S, Christiansen B, Gebel J, Goroncy-Bermes P, Hartemann P, et al. Antibiotic resistance: What is so special about multidrug-resistant Gram-negative bacteria? GMS Hyg Infect Control. 2017;12:Doc05.

2. Magiorakos AP, Srinivasan A, Carey RB, Carmeli Y, Falagas ME, Giske CG, et al. Multidrug-resistant, extensively drug resistant and pandrug-resistant bacteria: an international expert proposal for interim standard definitions for acquired resistance. Clin Microbiol Infect. 2012;18:268-81.

3. Kang Cl, Kim SH, Park WB, Lee KD, Kim HB, Kim EC, et al. Bloodstream infections caused by antibiotic-resistant gram-negative bacilli: risk factors for mortality and impact of inappropriate initial antimicrobial therapy on outcome. Antimicrob Agents Chemother. 2005;49(2):760-6.

4. Lockhart SR, Abramson MA, Beekmann SE, Gallagher G, Riedel S, Diekema DJ, et al. Antimicrobial resistance among Gram-negative bacilli causing infections in intensive care unit patients in the United States between 1993 and 2004. J Clin Microbiol. 2007;45(10):3352-9.

5. Parajuli NP, Acharya SP, Mishra SK, Parajuli K, Rijal BP, Pokhrel BM. High burden of antimicrobial resistance among gram negative bacteria causing healthcare associated infections in a critical care unit of Nepal. Antimicrob Resist Infect Control. 2017;6:67.

6. Falagas ME, Bliziotis IA, Kasiakou SK, Samonis G, Athanassopoulou P, Michalopoulos A. Outcome of infections due to pandrug-resistant (PDR) Gram-negative bacteria. BMC Infect Dis. 2005;5:24.

7. Druge S, Ruiz S, Vardon-Bounes F, Grare M, Labaste F, Seguin T, et al. Risk factors and the resistance mechanisms involved in Pseudomonas aeruginosa mutation in critically ill patients. J Intensive Care. 2019 Jul;19:7:36.

8. Kollef $\mathrm{MH}$. Broad-spectrum antimicrobials and the treatment of serious bacterial infections: getting it right up front. Clin Infect Dis. 2008;47(Suppl 1):3-13.

9. Micek ST, Welch EC, Khan J, Pervez M, Doherty JA, Reichley RM, et al. Empiric combination antibiotic therapy is associated with improved outcome against sepsis due to Gram-negative bacteria: a retrospective analysis. Antimicrob Agents Chemother. 2010;54(5):1742-8.

10. Zowawi HM. Antimicrobial resistance in Saudi Arabia. An urgent call for an immediate action. Saudi Med J. 2016;37(9):935-40. 
11. Boucher HW, Talbot GH, Bradley JS, Edwards JE, Gilbert D, Rice LB, et al. Bad bugs, no drugs: no ESKAPE! An update from the Infectious Diseases Society of America. Clin Infect Dis. 2009;48(1):112.

12. Cosgrove SE. The relationship between antimicrobial resistance and patient outcomes: mortality, length of hospital stay, and health care costs. Clin Infect Dis. 2006;42(Suppl 2):82-9.

13. Cosgrove SE. The relationship between antimicrobial resistance and patient outcomes: mortality, length of hospital stay, and health care costs. Clin Infect Dis. 2006;42:82-9.

14. Ben-David D, Kordevani R, Keller N, Tal I, Marzel A, Gal-Mor O, et al. Outcome of carbapenem resistant Klebsiella pneumoniae bloodstream infections. Clin Microbiol Infect. 2012;18(1):54-60.

15. Blot S, Vandewoude K, De Bacquer D, Colardyn F. Nosocomial bacteremia caused by antibioticresistant gram-negative bacteria in critically ill patients: clinical outcome and length of hospitalization. Clin Infect Dis. 2002;34(12):1600-6.

16. Menashe G, Borer A, Yagupsky P, Peled N, Gilad J, Fraser D, et al. Clinical significance and impact on mortality of ESBL-producing gram-negative isolates in nosocomial bacteremia. Scand J Infect Dis. 2001;33(3):188-93.

17. Kollef MH. Inadequate antimicrobial treatment: an important determinant of outcome for hospitalized patients. Clin Infect Dis. 2000; Suppl 4:S131-8.

18. Basak S, Singh P, Rajurkar M. Multidrug Resistant and Extensively Drug Resistant Bacteria: A Study. J Pathogens. 2016;2016:1-5.

19. Gupta V, Ye G, Olesky M, Lawrence K, Murray J, Yu K. National prevalence estimates for resistant Enterobacteriaceae and Acinetobacter species in hospitalized patients in the United States. Int $\mathrm{J}$ Infect Dis. 2019;85:203-11.

20. Alqasim A, Abu Jaffal A, Alyousef A. Prevalence of Multidrug Resistance and Extended-Spectrum $\beta$ Lactamase Carriage of Clinical Uropathogenic Escherichia coli Isolates in Riyadh, Saudi Arabia. Int J Microbiol. 2018;3026851.

21. Siwakoti S, Subedi A, Sharma A, Baral R, Bhattarai NR, Khanal B. Incidence and outcomes of multidrug-resistant gram-negative bacteria infections in intensive care unit from Nepal- a prospective cohort study. Antimicrob Resist Infect Control. 2018;7:114.

22. Gupta R, Malik A, Rizvi M, Ahmed M, Singh A, et al. Epidemiology of multidrug-resistant gramnegative pathogens isolated from ventilator-associated pneumonia in ICU patients. J Glob Antimicrob Resist. 2017;9:47-50.

23. World Health Organization (WHO). list-of-bacteria-for-which-new-antibiotics-are-urgently-needed. https://www.who.int/news-room/detail/27-02-2017-who-publishes-list-of-bacteria-for-which-newantibiotics-are-urgently-needed.

24. Coque TM, Baquero F, Canton R. Increasing prevalence of ESBL-producing Enterobacteriaceae in Europe. Euro Surveill. 2008;13:47.

25. Zhanel GG, DeCorby M, Laing N, Weshnoweski B, Vashisht R, Tailor F, et al. Antimicrobial-resistant pathogens in intensive care units in Canada: results of the Canadian National Intensive Care Unit 
(CAN-ICU) study, 2005-2006. Antimicrob Agents Chemother. 2008;52(4):1430-7.

26. Jamal W, Rotimi VO, Khodakhast F, Saleem R, Pazhoor A, Al Hashim G, et al. Prevalence of extendedspectrum $\beta$-lactamases in Enterobacteriaceae, Pseudomonas and Stenotrophomonas as determined by the VITEK 2 and E test systems in a Kuwait teaching hospital. Med Prin Pract. 2005;14:325-31.

27. Al-Zarouni M, Senok A, Rashid F, Al-Jesmi SM, Panigrahi D. Prevalence and antimicrobial susceptibility pattern of extended-spectrum $\beta$-lactamase-producing Enterobacteriaceae in the United Arab Emirates. Med Prin Pract. 2008;17:32-6.

28. Kandeel A. Prevalence and risk factors of extended-spectrum $\beta$-lactamases producing Enterobacteriaceae in a general hospital in Saudi Arabia. Journal of Microbiology Infectious Diseases. 2014;4(2):50-4.

29. Lai CC, Wu UI, Wang JT, Chang SC. Prevalence of carbapenemase-producing Enterobacteriaceae and its impact on clinical outcomes at a teaching hospital in Taiwan. JFMA. 2013;112(8):492-6.

30. El Wartiti MA, Bahmani FZ, Elouennass M, Benouda A. Prevalence of Carbapenemase-Producing Enterobacteriaceae in a University Hospital in Rabat, Morocco: A 19-Months Prospective Study. Int Arab J Antimicrob Agents. 2012;2(3:4):1-6.

31. Kandeel A. Epidemiology of carbapenemase producing Enterobacteriaceae in a general hospital. JMID. 2015;5(2):57-62.

32. Garbati M, Sakkijha H, Abushaheen A. Infections due to Carbapenem Resistant Enterobacteriaceae among Saudi Arabian Hospitalized Patients: A Matched Case-Control Study. Biomed Res Int. 2016;2016:3961684.

33. Alotaibi F, Bukhari E, Al-Mohizea M, Hafiz T, Essa EB, AlTokhais YI. Emergence of carbapenemresistant Enterobacteriaceae isolated from patients in a university hospital in Saudi Arabia. Epidemiology, clinical profiles and outcomes. J Infect Public Health. 2017;10(5):667-73.

34. Viceconte G, Maraolo AE, lula VD, Catania MR, Tosone G, Orlando R. Appropriateness of antibiotic prescription for targeted therapy of infections caused by multidrug-resistant bacteria: assessment of the most common improper uses in a tertiary hospital in southern Italy. Infez Med. 2017;25(3):22433.

35. Tzouvelekis LS, Markogiannakis A, Piperaki E, Souli M, Daikos GL. Treating infections caused by carbapenemase-producing Enterobacteriaceae. Clin Microbiol Infect. 2014;20(9):862-72.

36. Patolia S, Abate G, Patel N, Patolia S, Frey S. Risk factors and outcomes for multidrug- resistant Gram-negative bacilli bacteremia. Ther Adv Infect Dis. 2018;5(1):11-8.

37. Blot S, Vandewoude K, De Bacquer D, Colardyn F. Nosocomial bacteremia caused by antibioticresistant Gram-negative bacteria in critically ill patients: clinical outcome and length of hospitalization. Clin Infect Dis. 2002;34:1600-6.

\section{Tables}

Table 1. Demographics and Baseline Characteristics Based on Resistance Type 


\begin{tabular}{|c|c|c|c|c|}
\hline Characteristic & $\begin{array}{l}\text { Overall } \\
(n=138)\end{array}$ & $\begin{array}{l}\text { Alive } \\
(n=22 / 138)\end{array}$ & $\begin{array}{l}\text { Dead } \\
(n=116 / 138)\end{array}$ & $\begin{array}{l}\text { p- } \\
\text { value* }\end{array}$ \\
\hline Gender & & & & 0.004 \\
\hline Male & $64(46.4 \%)$ & $4(18.2 \%)$ & $60(51.7 \%)$ & \\
\hline Female & $74(53.6 \%)$ & $18(81.8 \%)$ & $56(48.3 \%)$ & \\
\hline Age & & & & 0.93 \\
\hline Mean \pm SD & $60.1 \pm 17.7$ & $59.7 \pm 21.4$ & $60.1 \pm 17.1$ & \\
\hline Median [min, max] & $\begin{array}{l}63.5[14.0, \\
94.0]\end{array}$ & $\begin{array}{l}69.5[15.0 \\
87.0]\end{array}$ & $63.0[14.0,94.0]$ & \\
\hline History of DM & $75(54.3 \%)$ & $12(54.5 \%)$ & $63(54.3 \%)$ & 0.98 \\
\hline History of cancer & $27(19.6 \%)$ & $5(22.7 \%)$ & $22(19.0 \%)$ & 0.68 \\
\hline Bacteria & & & & 0.26 \\
\hline E. coli & $71(51.4 \%)$ & $12(54.5 \%)$ & $59(50.9 \%)$ & \\
\hline K. pneumoniae & $45(32.6 \%)$ & $9(40.9 \%)$ & $36(31.0 \%)$ & \\
\hline Enterobacter species & $22(15.9 \%)$ & $1(4.5 \%)$ & $21(18.1 \%)$ & \\
\hline MDR/XDR strains & & & & 0.78 \\
\hline MDR & $130(94.2 \%)$ & $21(95.5 \%)$ & $109(94.0 \%)$ & \\
\hline XDR & $8(5.8 \%)$ & $1(4.5 \%)$ & $7(6.0 \%)$ & \\
\hline Resistance Type & & & & 0.48 \\
\hline ESBL & $71(51.4 \%)$ & $13(59.1 \%)$ & $58(50.0 \%)$ & \\
\hline CPE & $14(10.1 \%)$ & $3(13.6 \%)$ & $11(9.5 \%)$ & \\
\hline Other & $53(38.4 \%)$ & $6(27.3 \%)$ & $47(40.5 \%)$ & \\
\hline Source & & & & 0.56 \\
\hline Urine & $34(24.6 \%)$ & $7(31.8 \%)$ & $27(23.3 \%)$ & \\
\hline Wound & $16(11.6 \%)$ & $4(18.2 \%)$ & $12(10.3 \%)$ & \\
\hline Blood & $15(10.9 \%)$ & $0(0 \%)$ & $15(12.9 \%)$ & \\
\hline Sputum & $14(10.1 \%)$ & $4(18.2 \%)$ & $10(8.6 \%)$ & \\
\hline Tracheal aspirate & $12(8.7 \%)$ & $2(9.1 \%)$ & $10(8.6 \%)$ & \\
\hline Central line & $12(8.7 \%)$ & $3(13.6 \%)$ & $9(7.8 \%)$ & \\
\hline Urinary catheter & $8(5.8 \%)$ & $1(4.5 \%)$ & $7(6.0 \%)$ & \\
\hline
\end{tabular}




\begin{tabular}{|lllll|}
\hline Peritoneal fluid & $7(5.1 \%)$ & $1(4.5 \%)$ & $6(5.2 \%)$ & \\
\hline Tissue & $7(5.1 \%)$ & $0(0 \%)$ & $7(6.0 \%)$ & \\
\hline Others & $13(9.3)$ & $0(0 \%)$ & $13(11.2 \%)$ & \\
\hline ESRD & $85(61.6 \%)$ & $11(50.0 \%)$ & $74(63.8 \%)$ & 0.22 \\
\hline Prior 4 weeks Aantibiotic use & $26(18.8 \%)$ & $4(18.2 \%)$ & $22(19.0 \%)$ & 0.93 \\
\hline Prior 90 days Aantibiotic use & $99(71.7 \%)$ & $12(54.5 \%)$ & $87(75.0 \%)$ & 0.05 \\
\hline Prior 4 weeks Aantibiotic use & $110(79.7 \%)$ & $18(81.8 \%)$ & $92(79.3 \%)$ & 0.79 \\
\hline Inotropes & $99(71.7 \%)$ & $12(54.5 \%)$ & $87(75.0 \%)$ & 0.05 \\
\hline $\begin{array}{l}\text { ICU length of stay } \geq 72 \text { hours before } \\
\text { culture }\end{array}$ & $75(54.3 \%)$ & $8(36.4 \%)$ & $67(57.8 \%)$ & 0.06 \\
\hline
\end{tabular}

*Chi-square test was used to compare categorical data and independent sample t-test to compare continuous data.

Data presented as mean \pm standard deviation for continuous variables, or number and (percentages) for categorical variables.

CPE, Carbapenem producing Enterobacteriaceae; DM, diabetes mellitus; ESBL, Extended spectrum $\beta$ lactamase; ESRD, end-stage renal disease; ICU, intensive care unit; MDR, multidrug-resistant; SD, standard deviation; XDR, extensively drug-resistant.

Table 2. Clinical Outcomes of Study Subjects Divided by MDR/XDR Status

\begin{tabular}{|lllll|}
\hline Outcome & Overall $(\mathbf{n = 1 3 8 )}$ & MDR group $(\mathrm{n}=130)$ & XDR group $(\mathrm{n}=\mathbf{8})$ & p-value* \\
\hline ICU LOS in days mean \pm SD & $27.1 \pm 32.7$ & $25.8 \pm 31.9$ & $48.0 \pm 39.4$ & 0.06 \\
\hline Culture negative, $\mathbf{n}(\%)$ & $56(40.6 \%)$ & $52(40.0 \%)$ & $4(50.0 \%)$ & 0.58 \\
\hline Hospital mortality, $\mathbf{n}(\%)$ & $116(84.1 \%)$ & $109(83.8 \%)$ & $7(87.5 \%)$ & 0.78 \\
\hline
\end{tabular}

${ }^{*}$ Chi-square test was used to compare categorical data and independent sample t-test to compare continuous data.

Data presented as mean \pm standard deviation for continuous variables, or number and (percentages) for categorical variables. 
ICU, intensive care unit; LOS, length of stay; MDR, multidrug-resistant; SD, standard deviation; XDR, extensively drug-resistant.

Table 3. Characteristics Associated with Hospital Mortality in Study Subjects $(\mathrm{N}=138)$

\begin{tabular}{|c|c|}
\hline & $\begin{array}{l}\text { Unadjusted Analysis } \\
\text { OR (95\% Cl) }\end{array}$ \\
\hline Male gender & $4.82(1.68-17.47)$ \\
\hline Age (years) & $1.00(0.97-1.03)$ \\
\hline History of DM & $0.99(0.39-2.48)$ \\
\hline History of Cancer & $0.80(0.28-2.62)$ \\
\hline XDR Strain & $1.35(0.22-25.90)$ \\
\hline \multicolumn{2}{|l|}{ Bacteria } \\
\hline K. Pneumoniae & Reference \\
\hline E. coli & $1.22(0.46-3.20)$ \\
\hline Enterobacterspecies & $5.23(0.90-100.28)$ \\
\hline \multicolumn{2}{|l|}{ Resistance Type } \\
\hline ESBL & $0.57(0.19-1.56)$ \\
\hline CPE & $0.46(0.11-2.48)$ \\
\hline Other & Reference \\
\hline Ventilation & $1.76(0.70-4.46)$ \\
\hline ESRD & $1.05(0.35-3.92)$ \\
\hline Prior 90 days antibiotic use & $0.85(0.23-2.54)$ \\
\hline Prior 4 weeks antibiotic use & $2.5(0.96-6.41)$ \\
\hline Inotropes & $2.39(0.95-6.41)$ \\
\hline ICU length stay $\geq 72 \mathrm{hrs}$ until culture & $0.81(0.32-2.04)$ \\
\hline Appropriateness of therapy & $1.58(0.63-3.98)$ \\
\hline
\end{tabular}


$\mathrm{Cl}$, confidence interval; $\mathrm{CPE}$, Carbapenem producing Enterobacteriaceae; DM, diabetes mellitus; $\mathrm{ESBL}$, Extended spectrum $\beta$-lactamase; ESRD, end-stage renal disease; ICU, intensive care unit; OR, odds ratio, $\mathrm{SD}$, standard deviation; XDR, extensively drug-resistant.

Table 4. Characteristics Associated with Negative Cultures in Study Subjects $(\mathrm{N}=138)$

\begin{tabular}{|c|c|}
\hline & $\begin{array}{l}\text { Unadjusted Analysis } \\
\text { OR (95\% Cl) }\end{array}$ \\
\hline Male gender & $0.62(0.31-1.22)$ \\
\hline Age (years) & $1.00(0.98-1.02)$ \\
\hline History of DM & $0.74(0.37-1.47)$ \\
\hline History of Cancer & $1.47(0.62-3.44)$ \\
\hline XDR Strain & $1.5(0.34-6.60)$ \\
\hline \multicolumn{2}{|l|}{ Bacteria } \\
\hline K. Pneumoniae & Reference \\
\hline E. coli & $0.98(0.46-2.11)$ \\
\hline Enterobacterspecies & $1.25(0.44-3.52)$ \\
\hline \multicolumn{2}{|l|}{ Resistance Type } \\
\hline ESBL & $0.67(0.32-1.39)$ \\
\hline CPE & $2.35(0.71-8.54)$ \\
\hline Other & Reference \\
\hline Ventilation & $0.30(0.14-0.60)$ \\
\hline ESRD & $1.05(0.35-3.92)$ \\
\hline Prior 90 days Antibiotics use & $0.43(0.18-0.99)$ \\
\hline Prior 4 weeks Antibiotics use & $0.54(0.25-1.15)$ \\
\hline Inotropes & $0.60(0.29-1.16)$ \\
\hline ICU length stay $\geq 72 \mathrm{hrs}$ until culture & $1.19(0.60-2.36)$ \\
\hline Appropriateness of therapy & $0.67(0.33-1.33)$ \\
\hline
\end{tabular}

$\mathrm{Cl}$, confidence interval; CPE, Carbapenem producing Enterobacteriaceae; DM, diabetes mellitus; ESBL, Extended spectrum $\beta$-lactamase; ESRD, end-stage renal disease; ICU, intensive care unit; OR, odds ratio, 
SD, standard deviation; XDR, extensive drug-resistant.

Table 5. Characteristics Associated with LOS in Study Subjects $(\mathrm{N}=138)$

\begin{tabular}{|c|c|c|}
\hline & $\begin{array}{l}\text { Unadjusted Analysis } \\
\text { Coefficient ( } 95 \% \mathrm{Cl})\end{array}$ & $\begin{array}{l}\text { Adjusted Analysis } \\
\text { Coefficient }(95 \% \mathrm{Cl})\end{array}$ \\
\hline Male gender & $-3.33(-14.42-7.76)$ & - \\
\hline Age (years) & $-0.16(-0.47-0.18)$ & - \\
\hline History of DM & $7.83(-3.21-18.87)$ & - \\
\hline History of Cancer & $-10.40(-24.21-3.42)$ & - \\
\hline XDR Strain & $22.21(-1.12-45.54)^{\star}$ & $2.03(-19.28-23.33)$ \\
\hline \multicolumn{3}{|l|}{ Bacteria } \\
\hline K. Pneumoniae & Reference & Reference \\
\hline E. coli & $-14.69(-26.86-[-2.51]) \star$ & $-4.64(-15.80-6.51)$ \\
\hline Enterobacter species & $-0.18(-16.75-16.39)$ & $-3.62(-18.36-11.12)$ \\
\hline \multicolumn{3}{|l|}{ Resistance Type } \\
\hline ESBL & $-2.44(-14.24-9.36)$ & - \\
\hline CPE & $3.69(-16.43-23.81)$ & - \\
\hline Other & Reference & - \\
\hline Ventilation & $17.34(6.31-28.38) *$ & $11.49(1.02-21.97)^{\star \star}$ \\
\hline ESRD & $6.45(-7.86-20.76)$ & - \\
\hline Prior 90 days Antibiotics use & $11.51(-2.09-25.11)^{\star}$ & $-1.33(-13.67-11.01)$ \\
\hline Prior 4 weeks Antibiotics use & $3.53(-8.74-15.79)$ & - \\
\hline Inotropes & $-12.24(-23.16-[-1.32])$ & 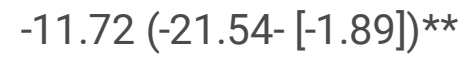 \\
\hline ICU length stay $\geq 72 \mathrm{hrs}$ until culture & $33.10(23.50-42.71)^{\star}$ & $28.65(17.85-39.46)^{\star \star}$ \\
\hline Appropriateness of therapy & $-7.71(-18.90-3.49)$ & - \\
\hline
\end{tabular}

* Variables with p-values below 0.1 were included in the adjusted model

** $p$-value $<0.05$ 
$\mathrm{Cl}$, confidence interval; $\mathrm{CPE}$, Carbapenem producing Enterobacteriaceae; DM, diabetes mellitus; $\mathrm{ESBL}$, Extended spectrum $\beta$-lactamase; ESRD, end-stage renal disease; ICU, intensive care unit; OR, odds ratio, SD, standard deviation; XDR, extensively drug-resistant .

\section{Figures}

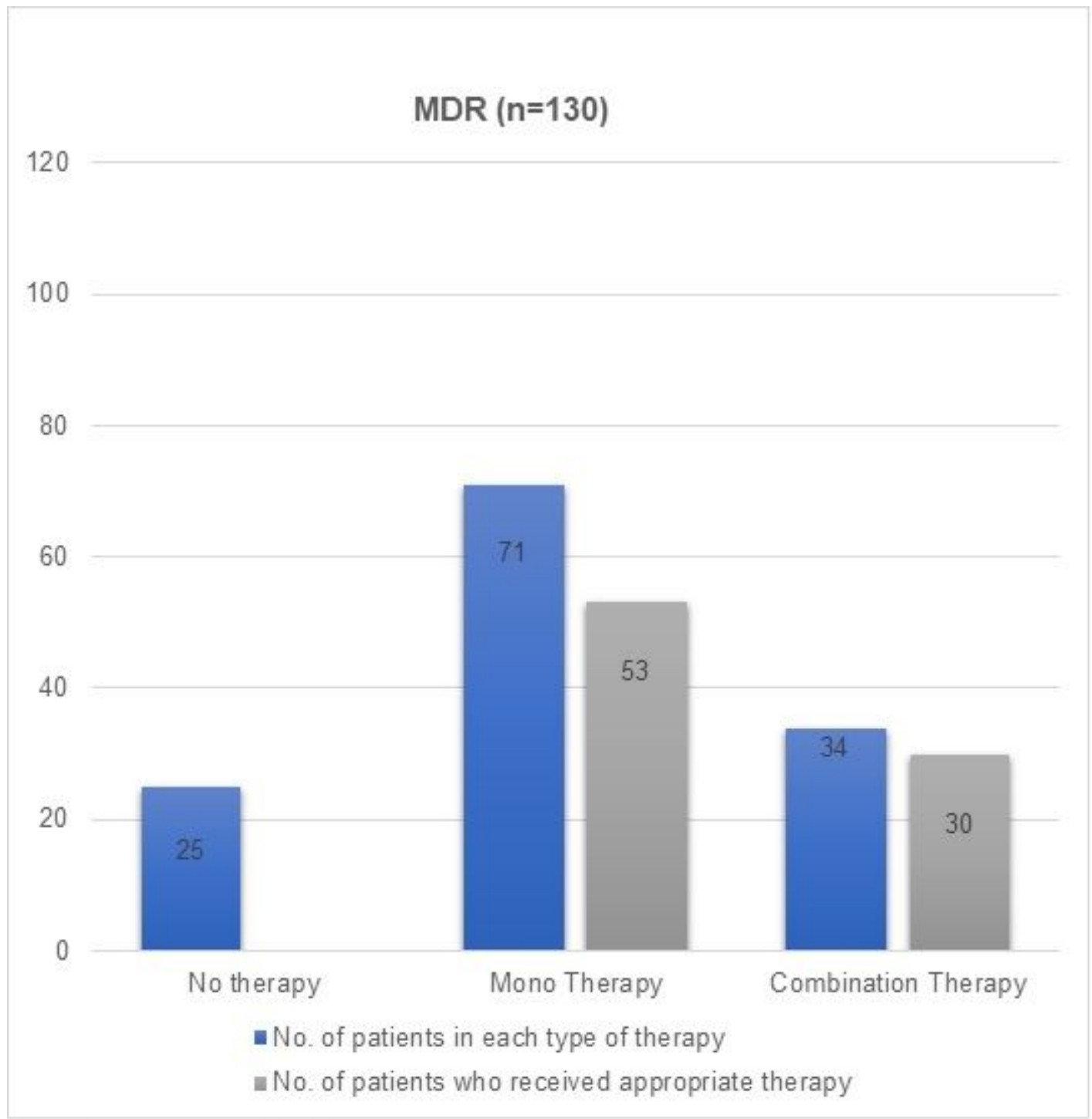

MDR, multidrug-resistant

\section{Figure 1}

Number of Antibiotic therapy and their Appropriateness in MDR Enterobacteriaceae Isolates 


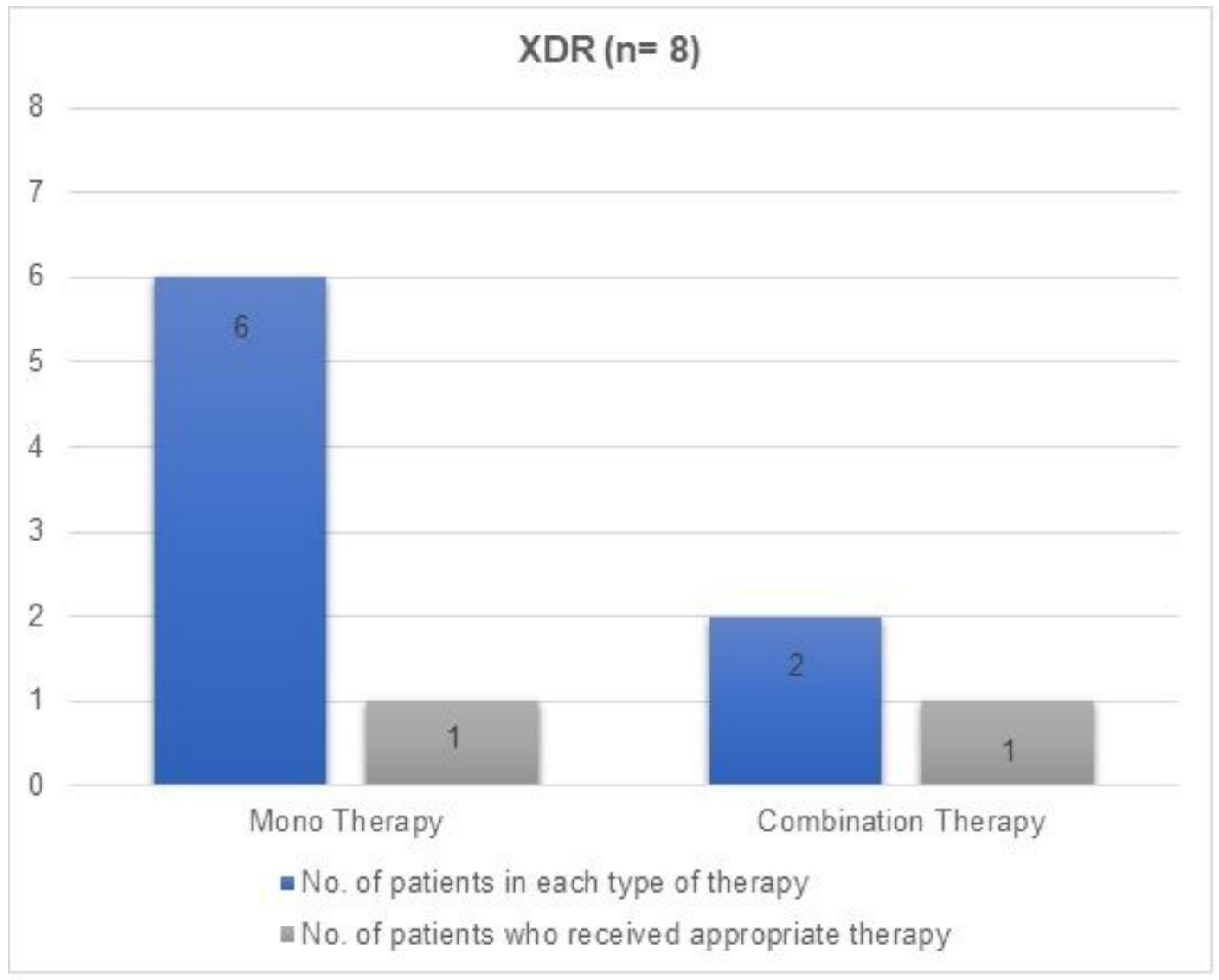

XDR, exten sively drug-resistant

\section{Figure 2}

Number of Antibiotic therapy and their Appropriateness in XDR Enterobacteriaceae Isolates

\section{Supplementary Files}

This is a list of supplementary files associated with this preprint. Click to download.

- SupplementaryMaterialMDRManuscript.docx 